\title{
The perceptual similarity of mirror-image acoustic patterns in speech
}

\author{
STEFANIE R. SHATTUCK \\ Cornell University, Ithaca, New York 14853
}

and

DENNIS H. KLATT

Research Laboratory of Electronics and Department of Electrical Engineering and Computer Science, Massachusetts Institute of Technology, Cambridge, Massachusetts 02139

\begin{abstract}
Prevocalic and postvocalic (unreleased) occurrences of a stop consonant differ in acoustic shape, but are not unrelated. In particular, the formant transitions taking place at release of a stop consonant approximately mirror in time the formant transitions occurring during closure, assuming that the vowel is the same. Several experiments have been performed using brief two-component tone burst approximations to the second and third formant transitions that occur in prevocalic and postvocalic allophones of $/ \mathrm{b}, \mathrm{d}, \mathrm{g} /$ in order to determine whether such mirror-image acoustic patterns are perceptually related. Listener judgments of similarity within triads of these stimuli indicate that mirror-image patterns representing the same place of articulation are less similar to each other than to patterns representing different places of articulation. Implications for the child who is acquiring language of the fact that mirror-image patterns in speech do not have inherent perceptual similarity are discussed.
\end{abstract}

Among the acoustic patterns which occur frequently in speech, certain pairs have an approximate mirror-image symmetry. For example, the formant transitions of a stop in prevocalic position mirror in time the formant transitions for the same stop in postvocalic position. Figure 1 illustrates the formant motions through time of the plosive consonants $/ b, d, g /$ in the context of vowel similar to $/ \varepsilon /$.

Only the three lowest formants are shown here. For $/ b /$, these formants fall in frequency during mouth closure (i.e., postvocalically). When the mouth is opened during release of the $/ b /$ (i.e., prevocalically), these three formants rise. For /d/ and $\mathrm{g} /$, the first formant motions follow this same pattern and thus do not contribute to decisions about place of articulation. However, the second and third formant motions do distinguish place of articulation. For $/ d /$, they generally rise during closure and fall during release, while for $/ g$ / they come together during closure and diverge on release. ${ }^{1}$

For each of the three plosives, the formant motions during closure very nearly mirror the formant motions during release. These formant transitions are important perceptual cues to place of articulation for plosive consonants (Liberman, Delattre, Cooper, \& Gerstman, 1954). In fact, they are the

This research was supported by the National Institute of Health (Grant NS-04332), by an NIH training grant (5-701-6M010 64), and by grants from the Grant Foundation and the Spencer Foundation to the Department of Psychology, M.I.T. primary cues for final unreleased plosives (Wang, 1959) and for nasals (Malecot, 1956). Yet, adults hear prevocalic and postvocalic instances of the same closure gesture as members of the same sound category, despite the radical mirror-image difference in their acoustic shape. How is this fact to be reconciled with the demonstrated importance of formant transitions for judgments of place of articulation?

Various explanations of this apparent contradiction have been proposed, including (1) reference to the shared articulatory gesture, (2) an association learned from listening to one's own VCV utterances, where postvocalic and prevocalic transitions are closely linked, and (3) the mirror-image acoustic relationship between the two sets of formant transitions. Mirror-image similarity is an important aspect of visual pattern similarity, and there seem to be many analogies between the functioning of the auditory and visual systems (Julesz \& Hirsh, 1972). The possibility arises that the mirror-image relationship is an important determinant of auditory pattern similarity as well.

This paper is addressed to the possibility that mirror-image acoustic patterns of the type shown in Figure 1 are intrinsically similar for a listener. If they are, then the acquisition of certain phonetic categories by the child learning a language would be considerably simplified. On the other hand, if there is no inherent perceptual similarity between mirrorimage sounds of this kind, then a child who has mastered a stop-vowel sequence like /ba/ will not 

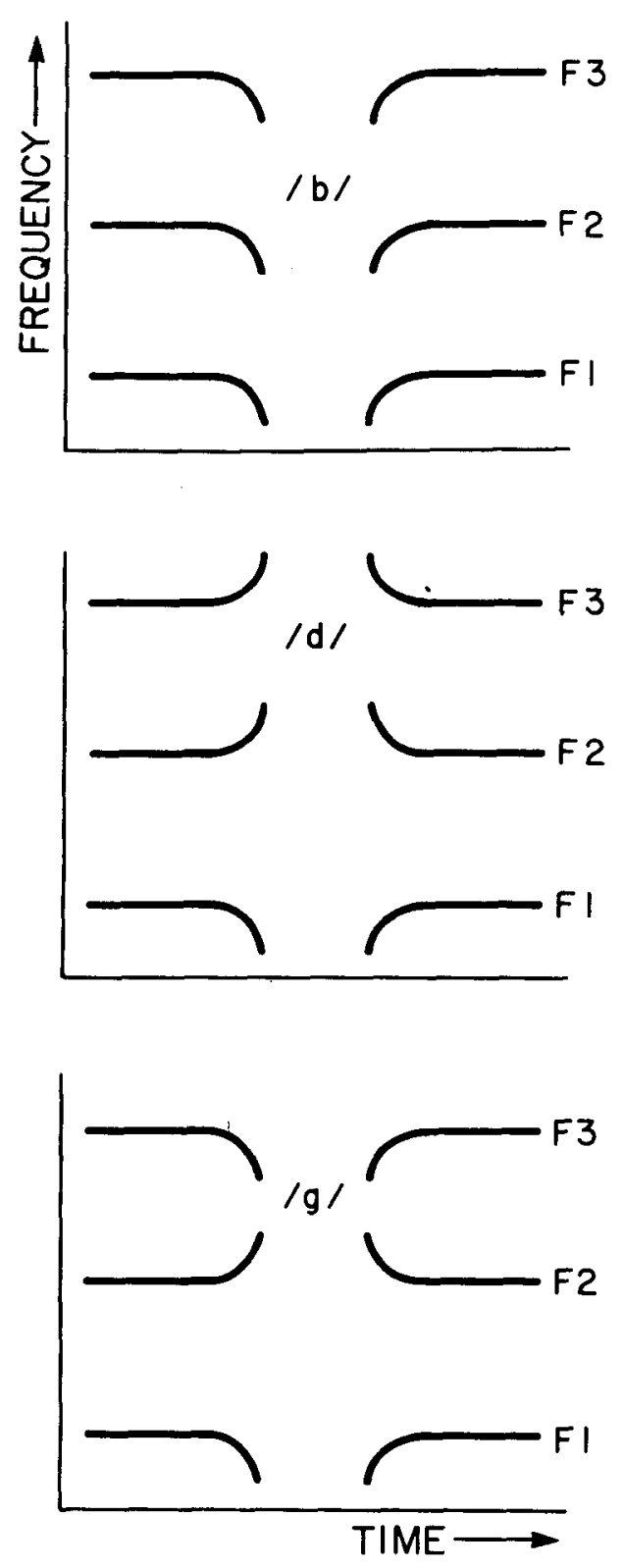

Figure 1. Idealizations of broad-band spectrograms illustrate the motions of the lowest three formant frequencies for intervocalic [b d g]. Frequency (from 0 to $3 \mathrm{kHz}$ ) is plotted on the vertical axis, and time (from 0 to $0.5 \mathrm{sec}$ ) is plotted on the horizontal axis. For each stop, the formant transitions that occur just prior to closure are approximately mirror images of transitions occurring after stop release.

automatically hear the corresponding mirror-image acoustic pattern for /ab/ as an instance of the same plosive consonant. In this case, postvocalic stops would have to be mastered using some other mechanism.

It is difficult to construct an experiment using natural speech stimuli to investigate the perceptual similarity of mirror-image acoustic patterns. We already know that adult listeners perceive prevocalic and postvocalic instances of a given stop as similar, and this bias, introduced by familiarity with English sound categories (acquired or innate), makes it difficult to use natural-speech results to support a mirror-image hypothesis.

In order to avoid the difficulties inherent in natural speech stimuli, we used stimuli composed of two brief pure tones with rapidly changing frequencies (Nabelek, Nabelek, \& Hirsh, 1970). These pure-tone glissandos are subjectively nonspeechlike, so that judgments of the similarity of mirror-image pairs are relatively uncolored by adult linguistic biases. In addition, pure-tone glissandos are very similar to formant transitions with respect to short-term spectral composition: both have a rapid spectral change such that the center frequency of the energy concentration shifts rapidly with time. While a formant transition and a glissando differ along other acoustic dimensions, ${ }^{2}$ this shared characteristic is probably the most important aspect of a formant transition for speech perception (Stevens, Note 1). Thus, two-glissando pure-tone acoustic patterns can be expected to tap a possible mechanism that is sensitive to mirror-image patterns in speech while avoiding the sound-category biases of adult speakers.

\section{EXPERIMENT 1}

\section{Method}

Subjects. The subjects were drawn from a pool of male and female students at M.I.T. who had volunteered to participate in paid experiments. They had no known hearing deficits.

Stimuli. In the first experiment, listeners compared brief synthetic glissando stimuli that resemble the rapid spectral changes in the second and third formants of initial and final $/ \mathrm{b}, \mathrm{d}, \mathrm{g} /$. A glissando corresponding to the first formant is not included in the synthetic stimuli because motions of the first formant do not differentiate between place of articulation for stops.

The stimulus ensemble is defined in Figure 2. Each stimulus consists of two 40-msec glissandos, i.e., brief tone bursts with a constant rate of frequency change throughout their duration. The tone bursts have $5-\mathrm{msec}$ linear rise and fall times. The amplitude of each glissando is adjusted to produce a 70-dB SPL output from a pair of binaural headphones. The frequency of a glissando component varies linearly about an average frequency $f$ by an amount $\Delta F$, which has been set equal to $350 \mathrm{~Hz}$ in all of the ex-

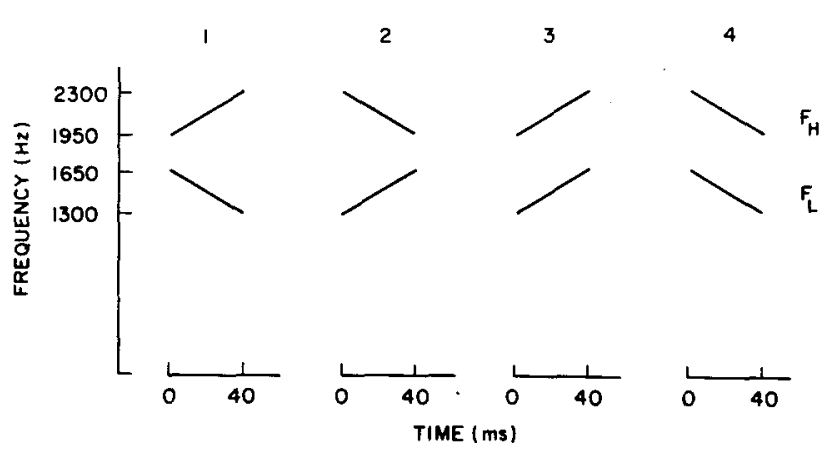

Figure 2. The frequency motions of a lower frequency glissando, $F_{L}$, and a higher frequency glissando, $F_{H}$, are defined for four stimuli that were used to elicit judgments of relative similarity in the first experiment. 


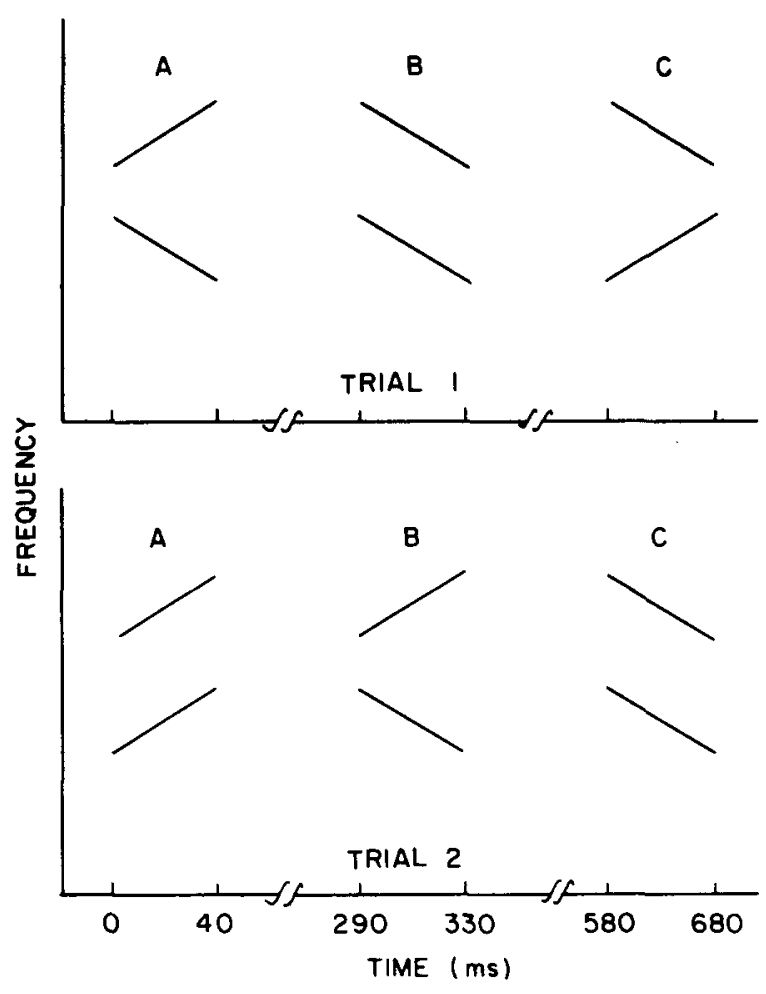

Figure 3. Examples are shown of two trials in the experiments involving judgments of relative similarity between three stimuli.

periments to be described. Three hundred and fifty hertz is a moderately large frequency motion for a stop-vowel transition.

The first stimulus in Figure 2 is an idealization of the motions of the second and third formants during the $\mathbf{4 0} \mathrm{msec}$ following release of a prevocalic $/ g /$ before a vowel such as $/ \Lambda /$. The second stimulus is a mirror-image of the first and corresponds to a postvocalic /g/ pattern. Stimulus 3 in Figure 2 resembles the second and third formant transitions for prevocalic $/ \mathrm{b} /$ and Stimulus 4 resembles postvocalic $/ b /$ if the vowel is a front vowel such as $/ I /$. Stimuli 3 and 4 also resemble second and third formant transitions for postrocalic and prevocalic $/ d /$ if the vowel is a back vowel such as $/ 3 /$ or $/ 2 /$.

A listening test using these four stimuli was generated by computer and recorded on analog tape for playback to a number of subjects. In each trial, three out of the four stimuli were presented with $250-\mathrm{msec}$ silent intervals between stimuli. Listeners were asked to indicate which of the three stimuli was the most different from the other two.

Figure 3 illustrates two sample trials from the experiment. If a listener judged Stimulus $B$ to be most dissimilar in either of the trials in Figure 3, he or she would be making a mirror-image response, since Stimuli $A$ and $C$ have no common acoustic attribute except that they are mirror images of one another. Stimuli A and B have one common glissando component, and Stimuli $B$ and $C$ also share a common glissando component. Thus, if a listener judges $\mathrm{C}$ or $\mathrm{A}$ to be the odd stimulus (i.e., finds it the most dissimilar of the three), he or she is not responding to mirror-image similarity. Instead, the judgement is based on the identical nature of either the higher or the lower component glissandos.

\section{Results}

The results of the perception test were consistent across 14 listeners. There were essentiallly no mirrorimage responses. Stimuli 1 and 4 of Figure 2 were always grouped together as most similar when compared with either 2 or 3 , and 2 and 3 were grouped together when compared with 1 or 4 . Stimuli 1 and 4 of Figure 2 have the same lower frequency glissando, although they differ with respect to the direction of frequency change of the higher frequency glissando component. Stimuli 2 and 3 also share a common lower-frequency glissando. Thus, the results of this initial experiment suggest that the lower frequency glissando component, corresponding to the second formant, dominates the percept.

\section{EXPERIMENT 2}

The results of the first experiment were discouraging for mirror-image similarity. However, the possibility remained that mirror-image responses might predominate if the amplitude of the lower frequency glissando were reduced so that it would no longer dominate the percept. Therefore, a second, larger, experiment was designed, to reveal the pattern of dissimilarity judgments when the amplitudes of the two glissando components were varied independently. In addition, three different frequency ranges were used instead of one, to determine whether (a) the predominance of the lower frequency glissando in Experiment 1 was restricted to a particular frequency range, or (b) the lower of the two components would always dominate. Six different intensity ratios and three different frequency ranges were used, for a total of 18 subtests.

\section{Method}

Each subtest consisted of 10 trials involving a set of four stimuli. Again, three stimuli from the set of four were presented in each trial, and listeners made a dissimilarity judgment. The frequencies of the glissando components for each of the stimulus sets are shown in Figure 4, and the glissando amplitudes were selected from a set of six relative sound pressure levels which differed in steps of $6 \mathrm{~dB}$, as shown on the ordinate of Figure 5.

Glissando components in Row $X$ of Figure 4 have average frequencies appropriate for the first and second formants; in Row $Z$, component frequencies approximate values appropriate for second and third formants, and Row Y provides an intermediate case. The results will determine whether it is always the lower frequency component which dominates, or whether a glissando in the specific lower frequency range of Experiment 1, corresponding to the $F_{2}$ range, will dominate no matter whether it is the highest or the lowest of the two components.

\section{Results}

Prior to listening to the experimental tape, subjects were given a 30-trial pretest to determine whether they were capable of making the required dissimilarity judgment when stimulus triads consist of only one glissando component each, i.e., when there is, in fact, acoustic identity between two of the three stimuli. Out of 18 listeners, 10 performed the task perfectly and 7 had scores ranging from $96 \%$ to $62 \%$ correct. One subject produced essentially random responses $(37 \%$ correct), and his data were discarded. 


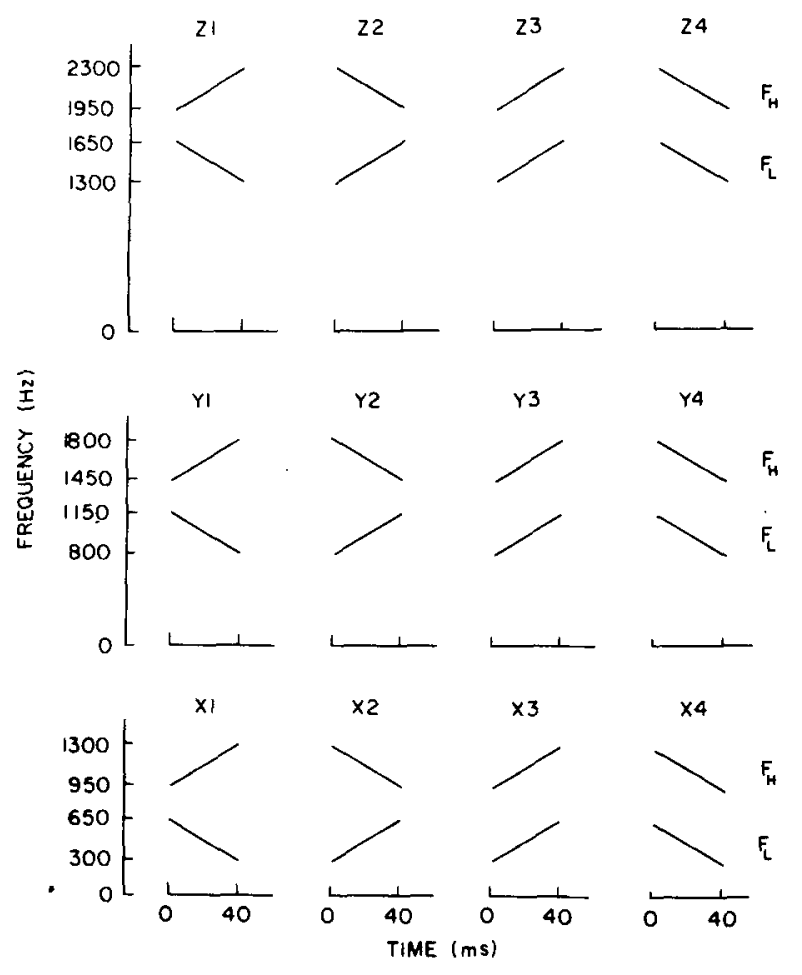

Figure 4. Each row defines the frequency motions of four stimuli in one of the subtests of Experiment 2.

Results from the remaining listeners for the twocomponents stimuli revealed that there were again very few mirror-image responses. The average responses of 17 listeners for one of the conditions in Experiment 2 are plotted in Figure 5; the few mirror-image responses will be discussed further below. In Figure 5, the horizontal axis corresponds to the relative amplitude levels of the two-glissando components, $A_{H} / A_{L}$, on the subtests involving a center frequency for the lower component of $F_{L}=475 \mathrm{~Hz}$, corresponding to Row $X$ in Figure 4 . The vertical axis indicates the percentage of trials on which the lower frequency components, the higher frequency components, or mirror-image symmetry of both components formed the basis of the similarity decision.

The value of $A_{H} / A_{L}$ for which the curves labeled "BASED ON $F_{L}$ " and "BASED ON $F_{H}$ " intersect will be called the equal perceptual importance crossover point. For $F_{L}=475 \mathrm{~Hz}$, this value was $-2 \mathrm{~dB}$. For $F_{L}=975 \mathrm{~Hz}$, Row $Y$ in Figure 4, the value increased substantially to $+13 \mathrm{~dB}$, and for $F_{\mathrm{L}}=$ $1,475 \mathrm{~Hz}$, Row $Z$ in Figure 4, the value increased again to $+26 \mathrm{~dB}$. The meaning of this change in crossover location is that there is a dramatic increase in the perceptual importance of the lower frequency glissando as it approaches the frequency region of the second formant (Klatt \& Shattuck, 1975; Klatt \& Shattuck-Hufnagel, Note 2). In other words, when the lower glissando component is in the frequency region of the second formant transition, the upper component must be much louder before it can influence similarity judgments. In contrast, when the higher of the two components is near the $F_{2}$ range, it does not have to be as loud in order to dominate the percept. Data from individual subjects are discussed in Klatt and Shattuck-Hufnagel (Note 2).

Mirror-image responses in Experiment 2. About 9\% of the responses in Experiment 2 were mirrorimage responses. A mirror-image response was more likely at amplitude ratios near the point where the curves of Figure 5 intersect. In these regions, mirrorimage responses approached $15 \%$. This is still less than the $33 \%$ figure that would be obtained if subjects were guessing randomly; evidently mirror-image responses are not preferred, even under conditions most conducive to their appearance.

Interestingly, however, not all the possible stimulus triads received an equal number of mirror-image responses. The first trial illustrated in Figure 3 is an example of a stimulus presentation that elicited most of the mirror-image responses, while the second trial in the same figure shows the kind of trial that elicited significantly fewer mirror-image responses. In more than $85 \%$ of the responses which reflected mirror-image similarity, listeners paired diverging with converging stimuli, as in A and C of Trial 1, Figure 3. The number of mirror-image responses to this type of triad approached $26 \%$ on subtests near the crossover point in Figure 5.

In contrast, the maximum number of mirror-image responses which paired rising-component stimuli with falling-component stimuli, as in $A$ and $C$ of Trial 2, Figure 3, was only $4 \%$. Apparently sets of rising glissandos are easy to distinguish from sets of falling glissandos, but if two of the stimuli in a triad contain both a rising and a falling component

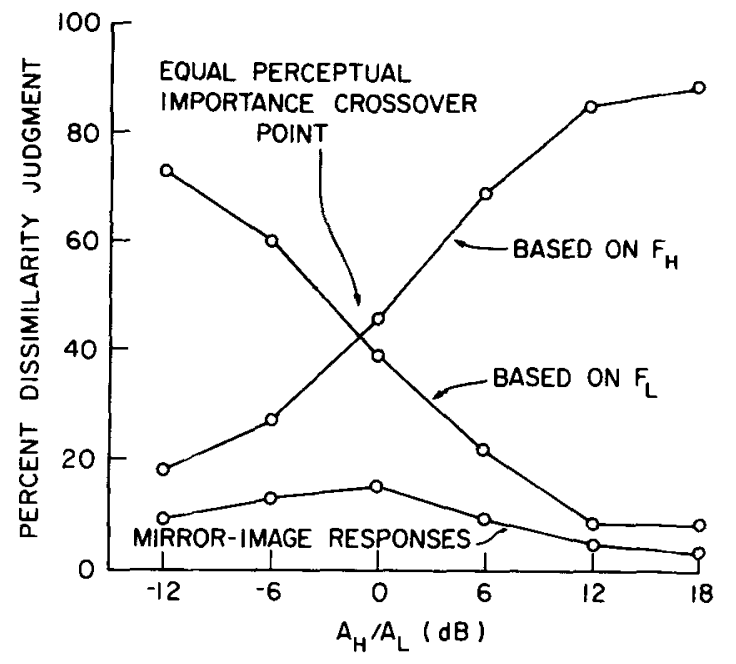

Figure 5. Average dissimilarity judgments based on the $F_{L}$ glissando compared with those based on the $F_{H}$ glissando component, and those based on mirror-image similarity, are plotted as a function of the relative amplitudes of the two components. 
of about equal perceptual importance, then similarity judgments become nearly random.

Overall, the results of the second experiment show conclusively that mirror-image acoustic patterns resembling formant transitions in stops are not perceptually similar for adults, particularly for amplitudes corresponding to natural speech.

\section{DISCUSSION}

Our results fail to support the hypothesis that once the child who is acquiring language has mastered a stop-vowel sequence such as $/ \mathrm{ba} /$, he or she automatically hears the corresponding mirrorimage acoustic pattern for the vowel-stop /ab/as an instance of the same category. Whatever the set of innate property detectors that the child is endowed with, we find no evidence in adults for acoustic detectors as complex as would be required to group together mirror-image rapid spectrum changes. This is consistent with the results of adaptation studies which show that the phonetic boundary between stimuli on a $/ \mathrm{ba} /-/ \mathrm{da} /$ continuum is not shifted in the expected direction by an /ab/ or /ad/ adaptor (Pisoni \& Tash, 1975; Ades, Note 3). If anything, the boundary is shifted in the opposite direction, a result which would be predicted on the basis of acoustic rather than phonetic adaptation.

It might be argued that stimuli constructed from brief pure-tone glissandos are not sufficiently speechlike to gain access to a set of innate phonetic detectors that group mirror-image formant patterns together. However, if such "limited-access" specialized phonetic detectors did exist, then the /ab/ adaptation experiments of Ades and of Pisoni and Tash should have had a different outcome, since they used speechlike sounds.

The process of discovering that the $/ b / b$ of $/ a b /$ is the same consonant as the $/ \mathrm{b} /$ of $/ \mathrm{ba} /$ may be mediated by articulatory similarity and acquired familiarity with the acoustic patterns for the intervocalic sequence $/ a b a /$. On the other hand, it is not inconceivable that a child remains unaware of the equivalence of these allophones of $/ b /$ until quite late in the language acquisition process, perhaps even until he or she learns to read.

Finally, the reasoning behind the present experiments is based on the hypothesis that rapid spectrum changes, i.e., formant transitions, are the primary perceptual cues for place of articulation of stops. A different decoding strategy is suggested by the observation that the spectrum at onset of a CV - is similar to the spectrum just at offset for the corresponding $-\mathrm{VC}$ and that these onset/offset spectra show a characteristic pattern for each place of articulation. This alternative decoding hypothesis has recently been revived by Stevens and Blumstein (1976). They note that onset and offset spectra have a compact energy distribution in the midfrequency region for velar stops, a concentration at higher frequencies for alveolar stops and a distribution over lower frequencies for labial stops. If listeners find acoustic invariance for the pace of articulation of stop consonants in the onset or offset spectra, rather than in the formant transition direction per se, then they would naturally group together pre- and postvocalic allophones of a given stop, without referring to the acoustic quality of the vowel.

\section{REFERENCE NOTES}

1. Stevens, K. N. The potential role of property detectors in the perception of consonants. Quarterly Progress Report No. 110, Research Laboratory of Electronics, M.I.T., 1973.

2. Klatt. D. H., \& Shattuck-Hufnagel, S. R. The perceptual importance of the second formant during rapid spectrum changes. Quarterly Progress Report No. 117. Research Laboratory of Electronics, M.I.T., 1976. Pp. 291-304.

3. Ades, A. E. Some effects of adaptation on speech perception. Quarterly Progress Report No. 111, Research Laboratory of Elec. tronics, M.I.T.. 1973. Pp.124-129.

\section{REFERENCES}

Julesz, B., \& Hirsh, I. J. Visual and auditory perception-an essay in comparison. In E. E. David \& P. B. Denes (Eds.), Human communication: $A$ unified view'. New York: McGrawHill. 1972. Pp. 283-340.

Klatt, D. H., \& Shattuck. S. R. Perception of brief stimuli that resemble formant transitions. In G. Fant \& M. A. A. Tatham (Eds.). A uditory analysis and perception of speech. New York: Academic Press, 1975. Pp. 294-301.

Liberman, A. M., Delattre, P., Cooper, F., \& Gerstman, L. The role of consonant-vowel transitions in the perception of the stop and nasal consonants. Psychological Monographs, 1954, 68 No. 8.

Malecot, A. Acoustic cues for nasal consonants. Language, 1956. 32. 274-284.

Nabelek, I. V., Nabelek, A. K., \& Hirsh, I. J. Pitch of tone bursts of changing frequency. Journal of the Acoustical Society of America, 1970, 48, 536-553.

PIsoni, D., \& TASH, J. Auditory property detectors and processing place features in stop consonants. Perception \& Psychophysics, $1975,18,401-408$.

Stevens, K. N., \& Blumstein, S. E. Context-independent properties for place of articulation in stop consonants. Journal of the Acoustical Society of America, 1976, 59, S40. (Supplement)

WANG, W. S.-Y. Transitions and release as perceptual cues for final plosives. Journal of Speech and Hearing Research. 1959, 2. 66-73.

\section{NOTES}

1. In order to bring out their visual mirror-image symmetry, these idealized representations of the acoustic patterns omit not only frequencies above $3,000 \mathrm{~Hz}$ but also the burst of frication noise that occurs at the release of a plosive. Bursts are known to contribute to the perception of place of articulation in prevocalic position, but they are not necessary for plosive identification (Liberman et al., 1954). We omitted them from our stimuli in the interests of a strong test of the mirrorimage hypothesis.

2. In a recent experiment, Klatt and Shattuck-Hufnagel (Note 2) successfully replicated the results of several of the experimental conditions described below, using formants in place of pure tone glissandos.

(Received for publication November 26, 1975; revision accepted July $6,1976$. ) 